\title{
Development of Nanovectors for the Targeted Delivery in Anopheles Mosquitoes of Drugs against Plasmodium Parasites
}

\author{
Elisabet Martí Coma-Cros ${ }^{1,2}$, Christian Grandfils ${ }^{3}$, Chantal Sevrin ${ }^{3}$, Jos Paulusse ${ }^{4}$, Naomi Hamelmann ${ }^{4}$, \\ Inga Siden-Kiamos ${ }^{5}$, John Vontas ${ }^{5}$, Lefteris Spanos ${ }^{5}$, Fatima Nogueira ${ }^{6}$, Henrique Silveira ${ }^{6}$, Sarah \\ Delacour $^{7}$, Luis Izquierdo ${ }^{2}$, Krijn Paaijmans ${ }^{2,8}$, Amedea Manfredi ${ }^{9}$, Paolo Ferruti ${ }^{9}$, Elisabetta Ranucci ${ }^{9}$, \\ Xavier Fernàndez-Busquets ${ }^{1,2}$ \\ ${ }^{1}$ Institute for Bioengineering of Catalonia, The Barcelona Institute of Science and Technology, Barcelona, Spain \\ elisabet.marti@isglobal.org; xfernandez_busquets@ub.edu \\ ${ }^{2}$ Barcelona Institute for Global Health (ISGlobal, Hospital Clínic-Universitat de Barcelona), Barcelona, Spain \\ luis.izquierdo@isglobal.org \\ ${ }^{3}$ Interfaculty Research Center of Biomaterials, University of Liège, Belgium \\ c.grandfils@uliege.be; csevrin@uliege.be \\ ${ }^{4}$ University of Twente, The Netherlands \\ j.m.j.paulusse@utwente.nl; n.m.hamelmann@utwente.nl \\ ${ }^{5}$ Institute of Molecular Biology and Biotechnology, FORTH, Heraklion, Greece \\ inga@imbb.forth.gr; vontas@imbb.forth.gr; spanos@imbb.forth.gr \\ ${ }^{6}$ Instituto de Higiene e Medicina Tropical, Universidade Nova de Lisboa, Portugal \\ nogueira.fatima@gmail.com; HSilveira@ihmt.unl.pt \\ ${ }^{7}$ School of Veterinary, University of Zaragoza, Spain \\ delacour@unizar.es \\ ${ }^{8}$ School of Life Sciences, Arizona State University, US \\ krijn.paaijmans@asu.edu \\ ${ }^{9}$ Dipartimento di Chimica, Università degli Studi di Milano, Italy \\ amedea.manfredi@unimi.it; paolo.ferruti@unimi.it; elisabetta.ranucci@unimi.it
}

\section{Extended Abstract}

Whereas nanomedical approaches to cure pathologies that are prevalent in high per capita income regions are intensively researched, there is an astonishing lack of nanomedicines being developed to treat the main cause of death in the impoverished world: infectious diseases, among which malaria is prominent. The unmet medical and patient need of malaria eradication will not be achieved unless the targeted delivery of new drugs is vastly improved. Encapsulation of drugs in targeted nanovectors is a rapidly growing area with a clear applicability to infectious disease treatment, and pharmaceutical nanotechnology has been identified as a potentially essential tool in the future fight against malaria. Polymers offer virtually unlimited diversity in chemistry, dimensions and topology, which renders them a class of materials that is particularly suitable for applications in nanoscale drug delivery strategies. The antimalarial drug curcumin encapsulated in different types of nanoparticles has shown in vitro activity against early mosquito stages of the GFP-expressing murine malaria parasite Plasmodium berghei. When females of the malaria mosquito vectors Anopheles atroparvus and Anopheles gambiae were fed with polymeric nanocarriers designed for the encapsulation of antimalarial drugs, the polymers were detected in the midgut lumen and in other insect's tissues, including the midgut epithelial cells. These results provide interesting perspectives for the direct administration of antimalarials to mosquitoes, targeting mosquito stages of Plasmodium.

This research was funded by (i) Fondazione Cariplo, Italy, grant number 2013-0584, (ii) Ministerio de Economía, Industria y Competitividad, Spain (which included FEDER funds), grant number PCIN-2017-100, and (iii) ERA-NET Cofund EURONANOMED, grant number 2017-178. 